Georgian Mathematical Journal

1(1994), No. 5, 561-565

\title{
COMPLEXITY OF THE DECIDABILITY OF THE UNQUANTIFIED SET THEORY WITH A RANK OPERATOR
}

\author{
M. TETRUASHVILI
}

\begin{abstract}
The unquantified set theory MLSR containing the symbols $\cup, \backslash,=, \in R(R(x)$ is interpreted as a rank of $x)$ is considered. It is proved that there exists an algorithm which for any formula $Q$ of the MLSR theory decides whether $Q$ is true or not using the space $c|Q|^{3}(|Q|$ is the length of $Q)$.
\end{abstract}

Let MLSR be an unquantified set theory whose language contains the symbols $\cup, \backslash,=, \in, R$, where $R$ denotes a unary functional symbol and $R(x)$ is interpreted as a rank of the set $x$ in the sense of J.von Neumann. The decidability problem for the theory MLSR reduces readily to testing the satisfiability of conjunctions of literals of the following types:

$$
(=) x=y \cup z, \quad x=y \backslash z, \quad(\in) \quad x \in y, \quad(R) \quad x=R(y)
$$

(the literal $x \notin y$ is equivalent to the formula $x \in z \& z=z \backslash y$, while the literal $x \neq y$ to the formula $[u \in x \& u \notin y] \vee[u \in y \& u \notin x]$, where $z, u$ are new variables). The conjunction $Q$ of literals is sometimes treated as a set of its literals.

Let, further, MLS be an unquantified set theory whose language contains the symbols $\cup, \backslash,=, \in$.

Definition 1. The interpretation $\alpha$ of the MLS language is called the singleton model of a formula $\varphi$ of this language if it associates a subset of the $\{\varnothing\}$ with each variable and $\varphi$ is true in $\alpha$.

Definition 2. The singleton model $\alpha$ of a set of all literals of the type $(=)$ of the formula $Q$ of the language of MLSR theory is called the place of the conjunction $Q$.

If $\alpha$ is the place of $Q$ and $y$ is the variable occurring in $Q$, then $y(\alpha)$ is the value of $y$ in $\alpha$.

1991 Mathematics Subject Classification. 03D15. 
Definition 3. Let $y$ be the variable occurring in $Q$. The place $\alpha$ of the formula $Q$ is called the place at $y$ if

$$
y(\alpha)= \begin{cases}1, & \text { when } x \in y \text { occurs in } Q \\ 0, & \text { when } x \notin y \text { occurs in } Q .\end{cases}
$$

D. Cantone et al. the following result have given in [1]:

Theorem 1. Let $Q$ be the conjunction of literals of the types $(=, \in, R)$ and $y_{1}, \ldots, y_{m}$ be its pairwise-distinct variables occurring in $Q$. Then $Q$ is satisfiable iff there exist

(i) a set $\Pi=\left\{\alpha_{1}, \ldots, \alpha_{n}\right\}$ of pairwise-distinct places of $Q$;

(ii) a function $F:\{1, \ldots, m\} \rightarrow\{1, \ldots, n\}$;

(iii) a set $W \subseteq\{1, \ldots, m\}$;

(iv) a sequence of integers $0=r_{0}<r_{1}<\cdots<r_{k}=n$ such that the following conditions are fulfilled:

(a) $\alpha_{F(i)}$ is the place at $y_{i}$ for all $i \in\{1, \ldots, m\}$;

(b) If $y_{i} \sim y_{j}$ (i.e., $\left.\forall \alpha \in \Pi\left(y_{i}(\alpha)=y_{j}(\alpha)\right)\right)$, then $F(i)=F(j) \quad(i, j \in$ $\{1, \ldots, m\})$;

(c) If $y_{i}\left(\alpha_{j}\right)=1$, then $k_{j}<k_{F(i)}(i \in\{1, \ldots, m\}, j \in\{1, \ldots, n\})$, where for every $j \in\{1, \ldots, n\} k_{j}$ denotes the number $r_{s}$ such that $r_{s-1}<j \leq r_{s}$;

(d) If $y_{j}=R\left(y_{i}\right)$ is a literal of $Q$, then $j \in W$;

(e) If $y_{j}=R\left(y_{i}\right)$ is a literal of $Q$, then $k_{i^{*}}=k_{j^{*}}$, where $h^{*}=\max \{l$ : $\left.y_{h}\left(\alpha_{l}\right)=1\right\}, h \in\{1, \ldots, m\}$

(f) $\forall i \in W \forall j \in W \quad\left(y_{i} \underset{\Pi}{\tilde{\pi}} y_{j} \Rightarrow y_{i} \widehat{\in} y_{j} \vee y_{j} \widehat{\in} y_{i}\right)$, where $y_{i} \widehat{\in} y_{j}$ denotes $y_{j}\left(\alpha_{F(i)}\right)=1$;

(g) $\forall i \in W \forall j \in W\left(y_{i} \widehat{\in} y_{j} \Rightarrow y_{i} \widehat{\subseteq} y_{j}\right)$, where $y_{i} \widehat{\subseteq} y_{j}$ denotes $\forall \alpha \in \Pi\left(y_{i}(\alpha)=\right.$ $\left.1 \Rightarrow y_{j}(\alpha)=1\right)$

(h) If for $i, j \in W$ there exists $h \in\{1, \ldots, n\}$ such that $y_{i}\left(\alpha_{h}\right)=0$ and $y_{j}\left(\alpha_{h}\right)=1$, then $\forall r \in\{1, \ldots, n\}\left(y_{i}\left(\alpha_{r}\right)=1 \Rightarrow k_{r}<k_{h}\right)$.

The proof of the necessity of this theorem makes essential use of the Venn diagram [2]. The construction of the set $\Pi$ is based on the Venn diagram for $m$ sets. Consequently the number $n$ of places is, in general, of order $2^{m}$. Therefore, despite the fact that Theorem 1 solves the decidability problem for the MLSR theory, the corresponding decision procedure has an exponential computational complexity (by space). Thus is of great interest to find an algorithm solving the decidability problem for the MLSR theory with a polynomial computational complexity (by space). The next theorem shows that such an algorithm can really be constructed.

Theorem 2. Let the conjunction $Q$ of literals of the types $(=, \in, R)$ is satisfiable and $y_{1}, \ldots, y_{m}$ be all pairwise-distinct variables of $Q$. Then there exist: 
(i) a set $\bar{\Pi}=\left\{\beta_{1}, \ldots, \beta_{\bar{n}}\right\}$ of pairwise-distinct places $Q$;

(ii) a function $\bar{F}:\{1, \ldots, m\} \rightarrow\{1, \ldots, \bar{n}\}$;

(iii) a set $W \subseteq\{1, \ldots, m\}$;

(iv) a sequence $0=\bar{r}_{0}<\bar{r}_{1}<\cdots<\bar{r}_{\bar{k}}=\bar{n}$ of natural numbers such that all the conditions (a)-(h) of Theorem 1 are fulfilled and $\bar{n} \leq c|Q|^{2}$, where $|Q|$ is the length of $Q$.

Proof. Let $Q$ be satisfiable. By virtue of Theorem 1 there exists: a set $\Pi=\left\{\alpha_{1}, \ldots, \alpha_{n}\right\}$, a function $F:\{1, \ldots, m\} \rightarrow\{1, \ldots, n\}$, a set $W$ and a sequence $r_{0}, r_{1}, \ldots, r_{k}$, satisfying the conditions (a)-(h) of this theorem.

Denote by $\Pi^{F}$ a set $\left\{\alpha_{F(i)}: i=1, \ldots, m\right\}$ of places of $Q . \Pi^{F} \subseteq \Pi$. Let $<i_{1}, j_{1}>, \ldots,<i_{\tau}, j_{\tau}>$ be pairs of numbers from $\{1, \ldots, m\}$ such that $i_{\nu}<j_{\nu}$ and $F\left(i_{\nu}\right) \neq F\left(j_{\nu}\right), \nu=1, \ldots, \tau$. Then by the condition (b) of Theorem $1 y_{i_{\nu}} \underset{\Pi}{\tilde{y}} y_{j_{\nu}}, \nu=1, \ldots, \tau$. Therefore for each pair $\left\langle i_{\nu}, j_{\nu}\right\rangle$, $\nu=1, \ldots, \tau$, there exists a place $\alpha_{l_{\nu}} \in \Pi$ with the lowest index $l_{\nu}$ such that $y_{i_{\nu}}\left(\alpha_{l_{\nu}}\right) \neq y_{j_{\nu}}\left(\alpha_{l_{\nu}}\right)$. Denote $\widetilde{\Pi}=\left\{\alpha_{l_{\nu}}: \nu=1, \ldots, \tau\right\}$. It is clear that $\tau \leq m(m-1) / 2$.

For each variable $y_{i}$ of $Q$ assume that $\alpha_{\mu_{i}}$ is a place from $\Pi$ with the highest index such that $y_{i}\left(\alpha_{\mu_{i}}\right)=1$ (if such a place exists in $\Pi$ ). Denote by $\Pi^{R}$ a set of all such places. Let $\Pi_{0}=\Pi^{F} \cup \widetilde{\Pi} \cup \Pi^{R}$. It is clear that $\operatorname{card}\left(\Pi_{0}\right) \leq\left(m^{2}+m\right) / 2$.

Finally, denote by $\Pi^{\Delta}$ a set of places from the set $\Pi$ which do not belong to the set $\Pi_{0}$ but whose indices are the ends of intervals $\left(r_{s-1}, r_{s}\right]$ containing the indices of places from the $\Pi_{0}$. Let $\bar{\Pi}=\Pi_{0} \cup \Pi^{\Delta}$. It is obvious that $\operatorname{card}\left(\Pi^{\Delta}\right) \leq \operatorname{card}\left(\Pi_{0}\right)$ and therefore $\operatorname{card}(\bar{\Pi}) \leq m^{2}+m$. Let $\bar{n}=\operatorname{card}(\bar{\Pi})$.

Let us now construct a new sequence of integers $0=\bar{r}_{0}<\bar{r}_{1}<\cdots<\bar{r}_{\bar{k}}=$ $\bar{n}$. Identify each place with its index $i$. From the set $\bar{\Pi}$ remove the integers which are not the indices of places from the set $\{1, \ldots, n\}$ and enumerate the elements of the set $\bar{\Pi}$, preserving their order by the natural numbers $1,2, \ldots, \bar{n}$. Clearly, in this case all numbers $r_{i}$ from the set $\bar{\Pi} \subseteq \Pi$ will acquire the corresponding values from the set $\{1, \ldots, \bar{n}\}$ which will form a new sequence $0=\bar{r}_{0}<\bar{r}_{1}<\cdots<\bar{r}_{\bar{k}}=\bar{n}$ of natural numbers.

Finally define the function $\bar{F}:\{1, \ldots, m\} \rightarrow\{1, \ldots, \bar{n}\}$ as follows: $\bar{F}(i)$ is the natural number from $[1, \bar{n}]$ into which the number $F(i)$ turns during the new enumeration of elements of the set $\bar{\Pi}(F(i)$ is the index of a place from $\bar{\Pi})$.

Let us check that the set $\bar{\Pi}=\left\{\beta_{1}, \ldots, \beta_{\bar{n}}\right\}$ of places of $Q$, the function $\bar{F}$, the set $W$ and the sequence $0=\bar{r}_{0}<\bar{r}_{1}<\cdots<\bar{r}_{\bar{k}}=\bar{n}$ satisfy all the conditions (a) $-(\mathrm{h})$ of Theorem 1 :

(a) $\beta_{\bar{F}(i)}$ is a place at $y_{i}, i=1, \ldots, m$, since $\beta_{\bar{F}(i)}=\alpha_{F(i)}$;

(b) If $y_{i} \sim y_{\bar{\Pi}}$, then on account of $\widetilde{\Pi} \subseteq \Pi_{0} \subseteq \bar{\Pi}$, we shall also have $y_{i} \sim y_{\Pi}$. But in that case $F(i)=F(j)$. Therefore $\bar{F}(i)=\bar{F}(j)$; 
(c) Let $y_{i}\left(\beta_{j}\right)=1$, i.e., $y_{i}\left(\alpha_{j^{\prime}}\right)=1$, where $j=1, \ldots, n$. Then $j$ and $F(i)$ belong to different intervals $\left(r_{s_{1}-1}, r_{s_{1}}\right]$ and $\left(r_{s_{2}-1}, r_{s_{2}}\right]$, respectively, the first interval preceding the second one, and, since $\alpha_{F(i)} \in \bar{\Pi}$ and $\alpha_{j^{\prime}}=$ $\beta_{j} \in \bar{\Pi}$, the ends of both intervals belong to the set of indices of places from $\bar{\Pi}$. But their order in the set $\{1, \ldots, n\}$ has remained unchanged during the new enumeration of elements of the set $\bar{\Pi}$, the numbers $j$ and $\bar{F}(i)$ belong to different new intervals corresponding to the sequence $\bar{r}_{0}, \bar{r}_{1}, \ldots, \bar{r}_{\bar{k}}$. Therefore $k_{j}<k_{\bar{F}(i)}, i \in\{1, \ldots, m\}, j \in\{1, \ldots, n\}$;

(d) It is obvious;

(e) Let $y_{j}=R\left(y_{i}\right)$ be a literal of the conjunction $Q$. Then by Theorem 1 $i^{*}$ and $j^{*}$ belong to the same interval $\left(r_{s-1}, r_{s}\right], s \leq n$. But $\Pi^{R} \subseteq \Pi_{0} \subseteq \Pi$ and the intervals were not subdivided during the transformation. Therefore, after the new enumeration the equality $k_{i^{*}}=k_{j^{*}}$ has also remained unchanged for the set $\bar{\Pi}$;

(f) Let $y_{i} \underset{\bar{\Pi}}{\widetilde{\Pi}} y_{j}$, i.e., $\exists \beta \in \bar{\Pi}\left[y_{i}(\beta) \neq y_{j}(\beta)\right]$. Since $\bar{\Pi} \subseteq \Pi$, we have $\exists \alpha \in \Pi\left[y_{i}(\alpha) \neq y_{j}(\alpha)\right]$, i.e. $y_{i} \underset{\Pi}{\sim} y_{j}$. For example, $y_{i} \widehat{\in} y_{j}$ is true by virtue of Theorem 1 (the case when $y_{j} \hat{\in} y_{i}$ is true is considered in a similar manner), i.e. $y_{j}\left(\alpha_{F(i)}\right)=1$. But $\alpha_{F(i)} \in \Pi^{F} \subseteq \bar{\Pi}$ and $\alpha_{F(i)}=\beta_{\bar{F}(i)}$. Therefore $y_{i} \widehat{\in} y_{j}$ is true in $\bar{\Pi}$;

(g) Let $y_{i} \widehat{\in} y_{j}$ hold in $\bar{\Pi}$, i.e. $y_{j}\left(\beta_{\bar{F}(i)}\right)=1$. But $\beta_{\bar{F}(i)}=\alpha_{F(i)}$, i.e. $y_{j}\left(\alpha_{F(i)}\right)=1$. Therefore $y_{i} \widehat{\in} y_{j}$ is true in $\Pi$. Then $y_{i} \widehat{\subseteq} y_{j}$ is true in $\Pi$ by virtue of Theorem 1, i.e. $\forall \alpha \in \Pi\left(y_{i}(\alpha)=1 \Rightarrow y_{j}(\alpha)=1\right)$. Since $\bar{\Pi} \subseteq \Pi$, the more so $\forall \alpha \in \bar{\Pi}\left(y_{i}(\alpha)=1 \Rightarrow y_{j}(\alpha)=1\right)$, i.e., $y_{i} \widehat{\subseteq} y_{j}$ is true in $\bar{\Pi}$;

(h) Let $i, j \in W$ and there exists $\bar{h} \in\{1, \ldots, \bar{n}\}$ such that $y_{i}\left(\beta_{\bar{h}}\right)=0$ and $y_{j}\left(\beta_{\bar{h}}\right)=1$. But $\beta_{\bar{h}}=\alpha_{h}$ for some $h \in\{1, \ldots, n\}$, i.e. $y_{i}\left(\alpha_{h}\right)=0$, and by Theorem $1 \forall r \in\{1, \ldots, n\}\left(y_{i}\left(\alpha_{r}\right)=1 \Rightarrow k_{r}<k_{h}\right)$. Let $\bar{r} \in\{1, \ldots, \bar{n}\}$. Then $\beta_{\bar{r}}=\alpha_{r}$ for some $r \in\{1, \ldots, n\}$. Therefore if $y_{i}\left(\beta_{\bar{r}}\right)=1$, then $y_{i}\left(\alpha_{r}\right)=1$. Consequently, $k_{r}<k_{h}$, i.e. $\alpha_{r}, \alpha_{h} \in \bar{\Pi}$, and $r$ and $h$ belong to different intervals $\left(r_{s_{1}-1}, r_{s_{1}}\right]$ and $\left(r_{s_{2}-1}, r_{s_{2}}\right]$, the first interval preceding the second one. The ends of these intervals were not removed from the set $\{1, \ldots, n\}$. Therefore the inequality $k_{\bar{r}}<k_{\bar{h}}$ has preserved.

Corrolary 1. There exists a Turing machine which for any formula $Q$ of the MLSR theory decides whether $Q$ is true or not, using the space $c|Q|^{3}$.

Let $\mathrm{MLSO}_{n}$ be an unquantified set theory whose language contains the symbols $\cup, \backslash,=, \in$ and a single-place predicate $O_{n}\left(O_{n}(x)\right.$ is interpreted as follows: $x$ is an ordinal). Since $O_{n}(x)$ iff $x=R(x)$, we have

Corrolary 2. There exists a Turing machine which for any formula $Q$ of the $\mathrm{MLSO}_{n}$ theory decides whether $Q$ is true or not, using the space $c|Q|^{3}$. 


\section{REFERENCES}

1. D. Cantone et al., Decision procedures for elementary sublanguages of set theory IV. Comm. Pure and Appl. Math. 40(1987), 37-77.

2. A. Kuzichev, Venn diagram. (Russian) Nauka, Moscow, 1968.

(Received 04.05.1993)

Author's address:

I. Vekua Institute of Applied Mathematics

of Tbilisi State University

2, University St., Tbilisi 380043

Republic of Georgia 